Bull. Austral. Math. Soc.

$20 \mathrm{G} 40,20 \mathrm{P} 05,15 \mathrm{~A} 52$

Vol. 64 (2001) [121-129]

\title{
A THEOREM ON POWER SERIES WITH APPLICATIONS TO CLASSICAL GROUPS OVER FINITE FIELDS
}

\begin{abstract}
ANDREW J. SPENCER
For some of the classical groups over finite fields it is possible to express the proportion of eigenvalue-free matrices in terms of generating functions. We prove a theorem on the monotonicity of the coefficients of powers of power series and apply this to the generating functions of the general linear, symplectic and orthogonal groups. This proves a conjecture on the monotonicity of the proportions of eigenvalue-free elements in these groups.
\end{abstract}

\section{INTRODUCTION}

In this paper we state and prove a result giving conditions for the coefficients of a power series raised to a power to decrease monotonically in size. This result has interesting consequences when used in conjunction with the results of Neumann and Praeger [1], on the proportion of eigenvalue-free matrices in the classical groups over finite fields.

We proceed as follows: Section 2 states and proves the main theorem of the paper; Section 3 introduces a function that was studied by Euler and states how this relates to the classical groups; Section 4 shows how we can use the techniques developed in Section 2 to work with the generating functions encountered in the previous section; Section 5 concludes with a result on the proportions of eigenvalue-free matrices in the general linear, symplectic and orthogonal groups over finite fields. Unless stated otherwise, all of our power series have real coefficients.

\section{A THEOREM ON POWER SERIES}

THEOREM 2.1. Suppose that $\lambda \in \mathbb{R}$ and $\lambda>1$. Let $A(z)=\sum_{n=0}^{\infty} a_{n} z^{n}$, where $a_{0}=1$ and $0<a_{n} \leqslant a_{n-1} / \lambda$ for $n \geqslant 1$. If $r$ is an integer such that $1 \leqslant r \leqslant \lambda$ and $a_{n}^{(r)}$ is the coefficient of $z^{n}$ in $(A(z))^{r}$ then

$$
0<a_{n}^{(r)}<a_{n-1}^{(r)} \leqslant 1
$$

for $n \geqslant 2$.

Received 30th October, 2000

Many thanks go to Dr Peter Neumann for his support and encouragement. I am also grateful to Duncan Brydon and the Engineering and Physical Sciences Research Council.

Copyright Clearance Centre, Inc. Serial-fee code: 0004-9727/01 \$A2.00+0.00. 
We postpone the proof as it relies on the following lemma.

LEMMA 2.2. Let $R(z)=\sum_{n=0}^{\infty} r_{n} z^{n}$ and $S(z)=\sum_{n=0}^{\infty} s_{n} z^{n}$ where all $r_{n}, s_{n}>0$ and the sequence $\left(r_{n}\right)_{n \geqslant 1}$ decreases strictly monotonically. Let $T(z)=R(z) S(z)$ and write $T(z)$ as $\sum_{n=0}^{\infty} t_{n} z^{n}$. For $n \geqslant 2$ if

$$
r_{0} s_{n}+r_{1} s_{n-1} \leqslant r_{0} s_{n-1}
$$

then $t_{n}<t_{n-1}$.

Proof: By definition,

$$
t_{n}=r_{0} s_{n}+r_{1} s_{n-1}+\cdots+r_{n} s_{0}
$$

and

$$
t_{n-1}=r_{0} s_{n-1}+r_{1} s_{n-2}+\cdots+r_{n-1} s_{0} .
$$

Since the coefficients of $R(z)$ are strictly montonically decreasing, for $2 \leqslant i \leqslant n$ we have $r_{i} s_{n-i}<r_{i-1} s_{n-i}$. The result follows.

Proof of TheOREM 2.1: Let $1 \leqslant r \leqslant \lambda$. We first deal with the coefficients $a_{0}^{(r)}$ and $a_{1}^{(r)}$. It is clear that $a_{0}^{(r)}=1$ and we can show by induction that $a_{1}^{(r)}=r a_{1}$. As $a_{1} \leqslant 1 / \lambda$ and $1 \leqslant r \leqslant \lambda$ we see that $a_{1}^{(r)} \leqslant 1$. Note that $a_{1}^{(r)}=1$ precisely when $r=\lambda$ and $a_{1}=1 / \lambda$.

We shall now use induction on $r$, up to $\lambda$, to show that for $1 \leqslant r \leqslant \lambda$ and for all $n \geqslant 2$, the inequality $a_{n}^{(r)}<a_{n-1}^{(r)}$ holds. For $r=1$ we are just considering the power series $A(z)$ for which the coefficients decrease strictly monotonically. Assume now that for some $r \leqslant \lambda-1$ and for all $n \geqslant 2$ we have $a_{n}^{(r)}<a_{n-1}^{(r)}$. We apply Lemma 2.2 with $R(z)=(A(z))^{r}$ and $S(z)=A(z)$. It follows that, for $n \geqslant 2$ if

$$
a_{0}^{(r)} a_{n}+a_{1}^{(r)} a_{n-1} \leqslant a_{0}^{(r)} a_{n-1}
$$

then

$$
a_{n}^{(r+1)}<a_{n-1}^{(r+1)} .
$$

Therefore showing that (1) holds would complete the inductive step. Now $a_{0}^{(r)}=1$ and $a_{1}^{(r)}=r a_{1}$. Furthermore, by assumption, $a_{n} \leqslant a_{n-1} / \lambda$, and $r \leqslant \lambda-1$, so

$$
a_{0}^{(r)} a_{n}+a_{1}^{(r)} a_{n-1} \leqslant \frac{1}{\lambda} a_{n-1}+r a_{1} a_{n-1} \leqslant a_{n-1}\left(\frac{1}{\lambda}+\frac{\lambda-1}{\lambda}\right)=a_{n-1} a_{0}^{(r)}
$$

as required. 
3. Generating functions Related to some Classical groups over finite FIELDS

We adopt the notation used by Neumann and Praeger in [1]. For a complex number $x$ with $|x|>1$ we define the function

$$
G(x ; z)=\prod_{i=1}^{\infty}\left(1-x^{-i} z\right) .
$$

It is shown in [1] that $G(x ; z)=\sum_{n=0}^{\infty} a_{n} z^{n}$ where $a_{0}=1$ and for $n \geqslant 1$,

$$
a_{n}=\frac{(-1)^{n}}{\prod_{i=1}^{n}\left(x^{i}-1\right)} .
$$

For $m \geqslant 1$, we shall be considering the classical groups $\operatorname{GL}(m, q), \operatorname{Sp}(2 m, q)$, $\mathrm{O}^{+}(2 m, q)$ and $\mathrm{O}^{-}(2 m, q)$ over the finite field $\mathbb{F}_{q}$. For $G \in\left\{\mathrm{GL}, \mathrm{Sp}, \mathrm{O}^{+}, \mathrm{O}^{-}\right\}$we define $v(G ; m, q)$ to be the proportion of eigenvalue-free matrices in the corresponding group of appropriate dimension. When dealing with the orthogonal groups we define

$$
v^{ \pm}(\mathrm{O} ; m, q)=v\left(\mathrm{O}^{+} ; m, q\right) \pm v\left(\mathrm{O}^{-} ; m, q\right)
$$

Considering these proportions as probabilities we define the associated generating functions

$$
V(G ; q, z)=1+\sum_{m=1}^{\infty} v(G ; m, q) z^{m}
$$

and

$$
V^{ \pm}(O ; q, z)=1+\sum_{m=1}^{\infty} v^{ \pm}(O ; m, q) z^{m}
$$

It follows that

$$
V\left(\mathrm{O}^{+} ; q, z\right)=\frac{1}{2}\left(V^{+}(\mathrm{O} ; q, z)+V^{-}(\mathrm{O} ; q, z)\right)
$$

and

$$
V\left(\mathrm{O}^{-} ; q, z\right)=\frac{1}{2}\left(V^{+}(\mathrm{O} ; q, z)-V^{-}(\mathrm{O} ; q, z)\right)
$$

The results in Table 1 are proved in [1], expressing the generating functions in terms of the function $G(x ; z)$.

\section{Results ON THE gENERATING FUNCTIONS}

For $G \in\left\{\mathrm{GL}, \mathrm{Sp}, \mathrm{O}^{+}, \mathrm{O}^{-}\right\}$we now have expressions for $V(G ; q, z)$ in the form

$$
V(G ; q, z)=(1-z)^{-1} \sum_{n=0}^{\infty} a_{n} z^{n} .
$$




\begin{tabular}{ll}
\hline Generating function & Related function \\
\hline \hline$V(\mathrm{GL} ; q, z)$ & $(1-z)^{-1} G(q ; z)^{q-1}$ \\
$V(\mathrm{Sp} ; q, z)$ & $(1-z)^{-1} G\left(q^{2} ; q z\right)^{2} G(q ; z)^{(q-3) / 2}, q$ odd \\
& $(1-z)^{-1} G\left(q^{2} ; q z\right) G(q ; z)^{(q-2) / 2}, q$ even \\
$V^{+}(\mathrm{O} ; q, z)$ & $(1-z)^{-1} G\left(q^{2} ; q z\right)^{2} G(q ; z)^{(q-3) / 2}, q$ odd \\
& $(1-z)^{-1} G\left(q^{2} ; q z\right) G(q ; z)^{(q-2) / 2}, q$ even \\
$V^{-}(\mathrm{O} ; q, z)$ & $G\left(q^{2} ; z\right)^{2} G(q ; z)^{(q-3) / 2}, q$ odd \\
& $G\left(q^{2} ; z\right) G(q ; z)^{(q-2) / 2}, q$ even \\
\hline
\end{tabular}

Table 1:

In this section we shall study these functions neglecting the factor $(1-z)^{-1}$ and prove results on the sequence $\left(a_{n}\right)_{n \geqslant 0}$. We say that the sequence $\left(a_{n}\right)_{n \geqslant n_{0}}$ is positive alternating if the sequence $\left((-1)^{n-n_{0}} a_{n}\right)_{n \geqslant n_{0}}$ has all terms greater than zero. We extend this definition to power series and define the class of positive alternating power series to be

$$
\mathcal{C}=\left\{A(z) \mid A(z)=\sum_{n=0}^{\infty}(-1)^{n} a_{n} z^{n}, a_{n}>0 \text { for all } n\right\} .
$$

It is not hard to show that $\mathcal{C}$ is closed under multiplication. For $q \geqslant 2$ the functions $G(q ; z), G\left(q^{2} ; q z\right)$ and $G\left(q^{2} ; z\right)$ all lie in $\mathcal{C}$ and it follows that any product of these must also lie in $\mathcal{C}$. In particular, from Table 1 , we see that $(1-z) V(\mathrm{GL} ; q, z),(1-z) V(\mathrm{Sp} ; q, z)$, $(1-z) V^{+}(\mathrm{O} ; q, z)$ and $V^{-}(\mathrm{O} ; q, z)$ all lie in $\mathcal{C}$.

If we have a positive alternating power series $A(z)=\sum_{n=0}^{\infty}(-1)^{n} a_{n} z^{n}$ where all $a_{n}>0$, then $A(-z)=\sum_{n=0}^{\infty} a_{n} z^{n}$. Hence to prove results on the monotonicity of the absolute value of the coefficients of $A(z)$ we can work with the coefficients of $A(-z)$ where all terms are positive.

TheOREM 4.1. Let $(1-z) V(\mathrm{GL} ; q, z)=\sum_{n=0}^{\infty}(-1)^{n} w_{n} z^{n}$. Then $w_{0}=w_{1}$ and the sequence $\left(w_{n}\right)_{n \geqslant 1}$ is strictly monotonically decreasing.

PROOF: Consider the power series $G(q ;-z)^{q-1}$. This is equal to $\sum_{n=0}^{\infty} w_{n} z^{n}$, where all $w_{n}>0$. It is clear that $w_{0}=1$ and induction on the power of $G(q ;-z)$ gives $w_{1}=1$. If $q=2$ then $G(q ;-z)^{q-1}=G(2 ;-z)$. In this case $\left(w_{n}\right)_{n \geqslant 1}$ is strictly monotonically decreasing and so we may assume that $q \geqslant 3$. Let $a_{n}$ be the coefficient of $z^{n}$ in $G(q ;-z)$. We know that for all $n \geqslant 1$,

$$
a_{n}=\frac{a_{n-1}}{q^{n}-1} \leqslant \frac{a_{n-1}}{q-1}
$$


and so we apply Theorem 2.1 to $G(q ;-z)$ with $\lambda=q-1$. This gives $w_{n}<w_{n-1}$ for all $n \geqslant 2$, as required.

THEOREM 4.2. Let $(1-z) V(\mathrm{Sp} ; q, z)=\sum_{n=0}^{\infty}(-1)^{n} w_{n} z^{n}$. Then the sequence $\left(w_{n}\right)_{n \geqslant 0}$ is strictly monotonically decreasing.

Proof: We just prove the case when $q \geqslant 3$ and $q$ is odd. A similar argument works for even $q$. We know that

$$
(1-z) V(\operatorname{Sp} ; q, z)=G\left(q^{2} ; q z\right)^{2} G(q ; z)^{(q-3) / 2} .
$$

We shall work with the function

$$
G\left(q^{2} ;-q z\right)^{2} G(q ;-z)^{(q-3) / 2}
$$

which has coefficients $\left(w_{n}\right)_{n \geqslant 0}$ that are all positive. We first show that the coefficients of $G\left(q^{2} ;-q z\right) G(q ;-z)^{(q-3) / 2}$ decrease strictly monotonically. If $q=3$ this is clear and we consider $q \geqslant 5$. Let

$$
A(z)=G(q ;-z)^{(q-3) / 2}=\sum_{n=0}^{\infty} a_{n} z^{n}, \quad B(z)=G\left(q^{2} ;-q z\right)=\sum_{n=0}^{\infty} b_{n} z^{n} .
$$

From Theorem 2.1 we know that the terms of the sequence $\left(a_{n}\right)_{n \geqslant 0}$ decrease strictly monotonically. We can use this together with Lemma 2.2 to see that $A(z) B(z)$ has coefficients which decrease strictly monotonically if, for $n \geqslant 1, a_{0} b_{n}+a_{1} b_{n-1}<a_{0} b_{n-1}$. Induction gives that

and we know that

$$
a_{1}=\frac{q-3}{2(q-1)}
$$

$$
b_{n}=b_{n-1} \frac{q}{q^{2 n}-1} \text {. }
$$

Therefore we need to show that

$$
\frac{q}{q^{2 n}-1}+\frac{q-3}{2(q-1)}<1
$$

This certainly holds for $n \geqslant 1$ and $q \geqslant 5$. Having proved that the coefficients of $G\left(q^{2} ;-q z\right) G(q ;-z)^{(q-3) / 2}$ are strictly monotonically decreasing we repeat the technique. This time let $A(z)=G\left(q^{2} ;-q z\right) G(q ;-z)^{(q-3) / 2}$ and $B(z)=G\left(q^{2} ;-q z\right)$, again with coefficients $\left(a_{n}\right)_{n \geqslant 0}$ and $\left(b_{n}\right)_{n \geqslant 0}$. Calculations give that $a_{0}=1$ and

$$
a_{1}=\frac{q}{q^{2}-1}+\frac{q-3}{2(q-1)} \text {. }
$$

Therefore to prove that $A(z) B(z)$ has coefficients that decrease strictly monotonically we must show that for $n \geqslant 1, a_{0} b_{n}+a_{1} b_{n-1}<a_{0} b_{n-1}$, that is,

$$
\frac{q}{q^{2 n}-1}+\frac{q}{q^{2}-1}+\frac{q-3}{2(q-1)}<1 \text {. }
$$


This can be seen to hold for all $q \geqslant 3$ and $n \geqslant 1$. The coefficients of $A(z) B(z)$ are precisely the sequence $\left(w_{n}\right)_{n \geqslant 0}$, and so the proof is complete.

THEOREM 4.3. Let $(1-z) V\left(\mathrm{O}^{+} ; q, z\right)=\sum_{n=0}^{\infty} w_{n} z^{n}$. Then the sequence $\left(w_{n}\right)_{n \geqslant 0}$ is positive alternating, $w_{0}=\left|w_{1}\right|=1$ and $\left(\left|w_{n}\right|\right)_{n \geqslant 1}$ decreases strictly monotonically. We omit the proof as it is similar to that of the next theorem.

THEOREM 4.4. Let $(1-z) V\left(\mathrm{O}^{-} ; q, z\right)=\sum_{n=0}^{\infty} w_{n} z^{n}$. Then the sequence $\left(w_{n}\right)_{n \geqslant 1}$ is positive alternating, $w_{0}=0$ and $\left(\left|w_{n}\right|\right)_{n \geqslant 1}$ decreases strictly monotonically.

Before we prove this we obtain some information about the power series $G\left(q^{2} ; z\right)^{2}$.

Lemмa 4.5. Let $a_{n}$ be the coefficient of $z^{n}$ in the power series $G\left(q^{2} ; z\right)^{2}$. For $q \geqslant 3$ and $n \geqslant 1$ we have

$$
\left|a_{n-1}\right|>q^{n}\left|a_{n}\right| \text {. }
$$

Proof: Let us denote the coefficient of $z^{n}$ in the power series $G\left(q^{2} ;-z\right)$ by $c_{n}$. It follows that

$$
\left|a_{n}\right|=\sum_{i=0}^{n} c_{i} c_{n-i} .
$$

Suppose that $n$ is odd and $n \geqslant 3$. Here we have

$$
\begin{aligned}
\left|a_{n}\right| & =2 c_{0} c_{n}+2 c_{1} c_{n-1}+\cdots+2 c_{(n-1) / 2} c_{(n+1) / 2} \\
\left|a_{n-1}\right| & =2 c_{0} c_{n-1}+2 c_{1} c_{n-2}+\cdots+2 c_{(n-3) / 2} c_{(n+1) / 2}+c_{(n-1) / 2} c_{(n-1) / 2} .
\end{aligned}
$$

We compare these equations term by term and claim that for $n \geqslant 3$ and $1 \leqslant i \leqslant(n+1) / 2$ we have $c_{n-i}>2 q^{n} c_{n-i+1}$. To prove this claim we note that

$$
c_{n-i+1}=\frac{c_{n-i}}{q^{2(n-i+1)}-1}
$$

and so

$$
c_{n-i}-2 q^{n} c_{n-i+1}=\left(1-\frac{2 q^{n}}{q^{2(n-i+1)}-1}\right) c_{n-i} .
$$

Now $c_{n-i}>0$ and for $i$ and $n$ in the range above,

$$
\frac{2 q^{n}}{q^{2(n-i+1)}-1}<1
$$

Hence $c_{n-i}-2 q^{n} c_{n-i+1}>0$ as required. The case when $n$ is even is similar and checking that $\left|a_{0}\right|>q\left|a_{1}\right|$ completes the proof.

Proof of Theorem 4.4: Suppose first that $q$ is even and $q \geqslant 2$. In this case

$$
V\left(\mathrm{O}^{-} ; q, z\right)=\frac{1}{2}(1-z)^{-1} G(q ; z)^{(q-2) / 2}\left(G\left(q^{2} ; q z\right)-(1-z) G\left(q^{2} ; z\right)\right) .
$$


Let $H(q ; z)=G\left(q^{2} ; q z\right)-(1-z) G\left(q^{2} ; z\right)$ with coefficients $\left(h_{n}\right)_{n \geqslant 0}$. It is not difficult to show that $h_{0}=0$ and for $n \geqslant 1$,

$$
h_{n}=(-1)^{n-1} \frac{q^{n}\left(q^{n}-1\right)}{\prod_{i=1}^{n}\left(q^{2 i}-1\right)} .
$$

Clearly $\left(h_{n}\right)_{n \geqslant 1}$ is positive alternating and for $n \geqslant 2$,

$$
\frac{\left|h_{n}\right|}{\left|h_{n-1}\right|}=\frac{q}{\left(q^{n}+1\right)\left(q^{n-1}-1\right)}<1
$$

telling us that the sequence $\left(\left|h_{n}\right|\right)_{n \geqslant 1}$ is strictly monotonically decreasing. Writing

$$
(1-z) V\left(\mathrm{O}^{-} ; q, z\right)=\frac{1}{2} G(q ; z)^{(q-2) / 2} H(q ; z)
$$

as $\sum_{n=0}^{\infty} w_{n} z^{n}$ we see that $w_{0}=0$ and $\left(w_{n}\right)_{n \geqslant 1}$ is positive alternating. If $q=2$ then $(1-z) V\left(\mathrm{O}^{-} ; q, z\right)=H(q ; z) / 2$, the coefficients of which satisfy the required monotonicity conditions. We may therefore assume that $q \geqslant 4$. We know from Theorem 2.1 that $G(q ;-z)^{(q-2) / 2}$ has coefficients that decrease strictly monotonically. With a little work we can apply Lemma 2.2 to $G(q ;-z)^{(q-2) / 2}$ and $-H(q ;-z)$ to see that the sequence $\left(\left|w_{n}\right|\right)_{n \geqslant 1}$ decreases strictly montonically.

Suppose now that $q \geqslant 3$ and $q$ is odd. Here

$$
V\left(\mathrm{O}^{-} ; q, z\right)=\frac{1}{2}(1-z)^{-1} G(q ; z)^{(q-3) / 2}\left(G\left(q^{2} ; q z\right)^{2}-(1-z) G\left(q^{2} ; z\right)^{2}\right) .
$$

Let $H(q ; z)=G\left(q^{2} ; q z\right)^{2}-(1-z) G\left(q^{2} ; z\right)^{2}$ with coefficients $\left(h_{n}\right)_{n \geqslant 0}$ and let $a_{n}$ be the coefficient of $z^{n}$ in $G\left(q^{2} ; z\right)^{2}$. It is clear that $h_{0}=0$ and for $n \geqslant 1$,

$$
h_{n}=q^{n} a_{n}-a_{n}+a_{n-1} .
$$

We know that the sequence $\left(a_{n}\right)_{n \geqslant 0}$ is positive alternating and for all $n \geqslant 1$, Lemma 4.5 tells us that $\left|a_{n-1}\right|>q^{n}\left|a_{n}\right|$. Therefore $h_{n}$ has the same sign as $a_{n-1}$ and

$$
\left|h_{n}\right|=\left|a_{n-1}\right|-\left(q^{n}-1\right)\left|a_{n}\right| .
$$

If $n \geqslant 2$, Equation 2 gives

$$
\left|h_{n-1}\right|-\left|h_{n}\right|=\left|a_{n-2}\right|-q^{n-1}\left|a_{n-1}\right|+\left(q^{n}-1\right)\left|a_{n}\right| .
$$

It is clear that $\left(q^{n}-1\right)\left|a_{n}\right|>0$ and Lemma 4.5 tells us that $\left|a_{n-2}\right|>q^{n-1}\left|a_{n-1}\right|$. Hence for all $n \geqslant 2,\left|h_{n}\right|<\left|h_{n-1}\right|$ and so $\left(\left|h_{n}\right|\right)_{n \geqslant 1}$ is strictly monotonically decreasing. This proves the theorem in the case $q=3$ as here $(1-z) V\left(\mathrm{O}^{-} ; q, z\right)=H(q ; z) / 2$. Assuming that $q \geqslant 5$, we consider the power series $G(q ; z)^{(q-3) / 2} H(q ; z)$. Since $h_{0}=0$, it is clear 
that the first coefficient in this power series is equal to zero, and after this the coefficients are positive alternating. Let us define

$$
F(z)=-H(q ;-z), \quad E(z)=G(q ;-z)=\sum_{n=0}^{\infty} e_{n} z^{n} .
$$

We shall work with $E(z)^{(q-3) / 2} F(z)$ as, neglecting sign, it has the same coefficients as $G(q ; z)^{(q-3) / 2} H(q ; z)$, that is $2(1-z) V\left(0^{-} ; q, z\right)$. Therefore, it remains to prove that $E(z)^{(q-3) / 2} F(z)$ has coefficients that, after the first, decrease strictly monotonically in size. For $0 \leqslant k \leqslant(q-3) / 2$ we define

$$
S^{(k)}(z)=E(z)^{k} F(z)
$$

and we denote its coefficients by $\left(s_{n}^{(k)}\right)_{n \geqslant 0}$. For $k \geqslant 0$ we see that

$$
s_{0}^{(k)}=0, \quad s_{1}^{(k)}=\frac{q-1}{q+1} \quad \text { and } s_{2}^{(k)}=\frac{1}{q^{2}+1}+\frac{k}{q+1} .
$$

We want to use induction on $k$ up to $(q-3) / 2$ to prove that the sequence $\left(s_{n}^{(k)}\right)_{n \geqslant 1}$ is strictly monotonically decreasing. By definition $S^{(0)}(z)=F(z)=-H(q ;-z)$ which we have already studied. Suppose that for some $k, 0 \leqslant k \leqslant(q-5) / 2$, the sequence $\left(s_{n}^{(k)}\right)_{n \geqslant 1}$ is strictly monotonically decreasing. We want to prove the condition for $S^{(k+1)}(z)$. An argument similar to that in the proof of Lemma 2.2 shows that for $n \geqslant 2$, if

$$
s_{0}^{(k)} e_{n}+s_{1}^{(k)} e_{n-1}+s_{2}^{(k)} e_{n-2}<s_{0}^{(k)} e_{n-1}+s_{1}^{(k)} e_{n-2}
$$

then $s_{n}^{(k+1)}<s_{n-1}^{(k+1)}$. Observing that $e_{n-1}=e_{n-2} /\left(q^{n-1}-1\right)$ and substituting the values of $s_{0}^{(k)}, s_{1}^{(k)}$, and $s_{2}^{(k)}$ we see that Equation 3 holds for $n$ and $k$ in the ranges specified. This completes the induction and the proof.

It is worth noting that we can find values of $q$ for which the coefficients of the power series $(1-z) V(\mathrm{U} ; q, z)$, associated with the unitary groups $\mathrm{U}(m, q)$, are neither alternating in sign nor monotonically decreasing in absolute value. In $[1$, p.579], there are a couple of mistakes. Firstly $G(-q ;-z)^{q+1} G\left(q^{2} ; z\right)^{-\left(q^{2}-q-2\right) / 2}$ is written in two places instead of $G(-q ;-z)^{q+1} G\left(q^{2} ; z\right)^{\left(q^{2}-q-2\right) / 2}$. Secondly the assertion is made that the coefficients of $(1-z) V(U ; q, z)$ alternate in sign for $q \geqslant 4$. As stated above, this is false.

\section{CONCLUding REMARKS}

From the fact that the generating function $V^{-}(O ; q, z) \in \mathcal{C}$, we deduce that for even $m$

$$
v\left(\mathrm{O}^{+} ; m, q\right)>v\left(\mathrm{O}^{-} ; m, q\right)
$$

and for odd $m$

$$
v\left(\mathrm{O}^{+} ; m, q\right)<v\left(\mathrm{O}^{-} ; m, q\right)
$$


Suppose that we fix a prime power $q$ and $G \in\left\{\mathrm{GL}, \mathrm{Sp}, \mathrm{O}^{+}, \mathrm{O}^{-}\right\}$. For $m \geqslant 1$ we define $v_{m}=v(G ; m, q)$. It is proved in [1] that these probabilities all tend to limits as $m \rightarrow \infty$ and we write $v_{\infty}=\lim _{m \rightarrow \infty} v_{m}$.

THEOREM 5.1. If $G \in\left\{\mathrm{GL}, \mathrm{Sp}, \mathrm{O}^{+}\right\}$then

$$
v_{2 m-1}<v_{2 m+1}<v_{\infty}<v_{2 m+2}<v_{2 m} .
$$

If $G=\mathrm{O}^{-}$then

$$
v_{2 m-1}>v_{2 m+1}>v_{\infty}>v_{2 m+2}>v_{2 m} .
$$

PROOF: Suppose that we write the generating function $V(G ; q, z)$ in the form

$$
V(G ; q, z)=(1-z)^{-1} \sum_{n=0}^{\infty} w_{n} z^{n}
$$

then for all $m \geqslant 1$,

$$
v_{m}=\sum_{i=0}^{m} w_{i}
$$

Furthermore, if the sequence $\left(w_{n}\right)_{n \geqslant 0}$ is positive alternating, the terms of $\left(w_{n}\right)_{n \geqslant 1}$ decrease strictly monotonically in absolute value, and the series $\sum w_{n}$ converges, then

$$
v_{2 m-1}<v_{2 m+1}<v_{\infty}<v_{2 m+2}<v_{2 m} .
$$

This is the situation when $G \in\left\{\mathrm{GL}, \mathrm{Sp}, \mathrm{O}^{+}\right\}$. When $G=\mathrm{O}^{-}$the inequalities are reversed since expressing $V\left(\mathrm{O}^{-} ; q, z\right)$ in the form (4) yields a sequence $\left(w_{n}\right)_{n \geqslant 0}$ that is positive alternating and whose terms decrease strictly monotonically in absolute value only after the first term $\left(w_{0}=0\right)$ has been removed.

It would be interesting to see a combinatorial explanation for these inequalities.

\section{REFERENCES}

[1] P.M. Neumann and C.E. Praeger, 'Derangements and eigenvalue-free elements in finite classical groups', J. London Math. Soc. (2) 58 (1998), 564-586.

St. Hugh's College

Oxford OX2 6LE

United Kingdom 